\title{
ON THE STOKES EQUATION WITH NEUMANN BOUNDARY CONDITION
}

\author{
YOSHIHIRO SHIBATA \\ Department of Mathematical Sciences, Waseda University \\ Ohkubo 3-4-1, Shunjuku-ku, Tokyo 169-8555, Japan \\ E-mail: yshibata@waseda.jp \\ SENJO SHIMIZU \\ Faculty of Engineering, Shizuoka University, Hamamatsu 432-8561, Japan \\ E-mail: tssshim@ipc.shizuoka.ac.jp
}

\begin{abstract}
In this paper, we study the nonstationary Stokes equation with Neumann boundary condition in a bounded or an exterior domain in $\mathbb{R}^{n}$, which is the linearized model problem of the free boundary value problem. Mainly, we prove $L_{p}-L_{q}$ estimates for the semigroup of the Stokes operator. Comparing with the non-slip boundary condition case, we have the better decay estimate for the gradient of the semigroup in the exterior domain case because of the null force at the boundary.
\end{abstract}

1. Introduction. Let $\Omega$ be a bounded or an exterior domain in $\mathbb{R}^{n}(n \geq 2)$ with boundary $\partial \Omega$ which is a $C^{2,1}$ hypersurface. We consider the nonstationary Stokes problem with Neumann boundary condition:

$$
\begin{cases}\partial_{t} u-\operatorname{Div} \mathrm{T}(u, \pi)=0, & \operatorname{div} u=0 \\ \mathrm{~T}(u, \pi) \nu=0 & \text { in } \Omega, t>0 \\ \left.u\right|_{t=0}=u_{0} & \text { on } \partial \Omega, t>0\end{cases}
$$

where $u$ is the unknown velocity vector, $\pi$ is the unknown pressure, and $u_{0}$ is a given

2000 Mathematics Subject Classification: 76D07, 35Q30.

Key words and phrases: Stokes equation, Neumann boundary condition, free boundary value problem, Stokes semigroup, $L_{p}$ estimates, $L_{p}-L_{q}$ estimates.

Research of the first author partly supported by Grant-in-Aid for Scientific Research (B)15340204, Ministry of Education, Sciences, Sports and Culture, Japan.

Research of the second author partly supported by Grant-in-Aid for Scientific Research (C)-14540171, Ministry of Education, Sciences, Sports and Culture, Japan.

The paper is in final form and no version of it will be published elsewhere. 
velocity vector. $\mathrm{T}$ is the stress tensor whose $(j, k)$ component is given by

$$
\begin{gathered}
\mathrm{T}_{j k}(u, \pi)=D_{j k}(u)-\delta_{j k} \pi, \quad j, k=1, \ldots, n, \\
D_{j k}(u)=\partial u_{j} / \partial x_{k}+\partial u_{k} / \partial x_{j}, \quad \delta_{j k}=1(j=k),=0(j \neq k) .
\end{gathered}
$$

For simplicity, we assume that the viscous coefficient $\mu=1$. Under the condition div $u=$ $0, \operatorname{Div} \mathrm{T}(u, \pi)=\Delta u-\nabla \pi$.

(1.1) is a model problem of the free boundary value problem (cf. Solonnikov [16] and Abels [1]). Let us consider the region $\Omega(t) \in \mathbb{R}^{n}$ occupied by the fluid which is given only at the initial time $t=0$, while for $t>0$ it is to be determined. In this model the effect of surface tension is neglected.

$$
\begin{cases}\partial_{t} v+(v \cdot \nabla) v-\Delta v+\nabla q=f(x, t) & \text { in } \Omega(t), t>0 \\ \nabla \cdot v=0 & \text { in } \Omega(t), t>0 \\ \mathrm{~T}(v, q) \nu_{t}+p_{0}(x, t) \nu_{t}=0 & \text { on } \partial \Omega(t), t>0 \\ \left.v\right|_{t=0}=v_{0} & \text { in } \Omega(0),\end{cases}
$$

where $\nu_{t}$ is the unit outer normal to $\partial \Omega(t)$ at the point $x, v_{0}$ is a given initial velocity, $\Omega(0)$ is the initial domain filled by the fluid, and $f(x, t)$ and $p_{0}(x, t)$ are the external mass force vector and the pressure defined on the whole space. Below we assume that $p_{0}(x, t)=0$, since we can arrive at this case by replacing $p(x, t)$ by $p+p_{0}$.

Following the approach due to Solonnikov [16], we reduce (1.2) to the problem as an initial boundary value problem in the given region $\Omega(0)=\Omega$. A kinematic condition for $\partial \Omega(t)$ is satisfied, which gives $\partial \Omega(t)$ as a set of points $x=x(\xi, t), \xi \in \partial \Omega$, where $x(\xi, t)$ is the solution of the Cauchy problem

$$
\frac{d x}{d t}=v(x, t),\left.\quad x\right|_{t=0}=\xi
$$

We can rewrite (1.2) as an initial boundary value problem in $\Omega$, if we go over the Euler coordinates $x \in \Omega(t)$ to the Lagrange coordinates $\xi \in \Omega$ connected with $x$ by (1.3). If a velocity vector field $u(\xi, t)$ is known as a function of the Lagrange coordinates $\xi$, then this connection can be written in the form

$$
x=\xi+\int_{0}^{t} u(\xi, \tau) d \tau:=X_{u}(\xi, t) .
$$

Passing to the Lagrange coordinates in (1.2) and setting $v\left(X_{u}(\xi, t), t\right)=u(\xi, t)$ and $\tilde{q}\left(X_{u}(\xi, t), t\right)=\pi(\xi, t)$, we obtain

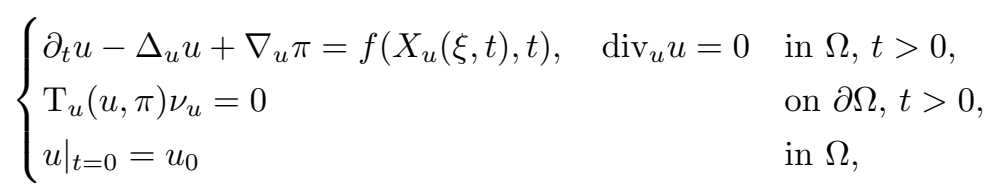

where using $A(u)={ }^{t}\left(D_{\xi} X_{u}\right)^{-1}(\xi, t)$,

$$
\begin{aligned}
& \nabla_{u}=A(u) \nabla, \operatorname{div}_{u} u=\nabla_{u} \cdot u=\operatorname{tr}(A(u) \nabla u), \\
& \Delta_{u}=\operatorname{div}_{u} \nabla_{u}, \nu_{u} \cdot T_{u}(u, \pi)=\nu_{u} \cdot\left(\nabla_{u} u+{ }^{t}\left(\nabla_{u} u\right)\right)-\pi \nu_{n}, \\
& \nu_{u}(\xi, t)=A(u) \nu_{\xi} /\left|A(u) \nu_{\xi}\right|
\end{aligned}
$$


$\nu_{\xi}$ denotes the unit outer normal at $\xi \in \partial \Omega$. If $t$ is small, then the operators $\Delta_{u}, \nabla_{u}, \operatorname{div}_{u}$ and $T_{u}$ are closed to $\Delta, \nabla$, div and $T$. Therefore we write (1.4) as a fixed point problem:

$$
\begin{cases}\partial_{t} u-\mu \Delta u+\nabla \pi=-\mu\left(\Delta-\Delta_{u}\right) u & \\ \quad+\left(\nabla-\nabla_{u}\right) \pi+f\left(X_{u}(\xi, t), t\right) & \text { in } \Omega, t>0, \\ \operatorname{div} u=\left(\operatorname{div}-\operatorname{div}_{u}\right) u & \text { in } \Omega, t>0, \\ T(u, \pi) \nu=\left(T \nu-T_{u} \nu_{u}\right)(u, \pi) & \text { on } \partial \Omega, t>0, \\ \left.u\right|_{t=0}=u_{0} & \text { in } \Omega .\end{cases}
$$

Our final goal is to prove a globally in time existence of solutions of (1.2) for small initial data by using the analytic semigroup approach. To do this, we have the following plan of analysis:

$1^{\circ}$ Analysis of the resolvent problem corresponding to (1.1).

$2^{\circ}$ Analytic semigroup approach to (1.1).

$3^{\circ} \quad L_{p}-L_{q}$ estimate of (1.1).

$4^{\circ}$ Maximal regularity of the linearized problem with inhomogeneous right members.

In this paper, we report on the results about $1^{\circ}, 2^{\circ}$ and $3^{\circ}$.

The free boundary value problem (1.2) was already solved by Solonnikov [16] in the bounded domain case. The linear problem (1.1) was already studied by using the theory of pseudo-differential operators with parameter (cf. Grubb and Solonnikov [10] and Grubb [8] and [9]). Our approach is completely different from [16], [10], [8] and [9].

2. Analysis of the resolvent problem to (1.1). The resolvent problem corresponding to $(1.1)$ is:

$$
\left\{\begin{array}{c}
\lambda u-\Delta u+\nabla \pi=f, \quad \operatorname{div} u=0 \quad \text { in } \Omega, \\
\left.\mathrm{T}(u, \pi) \nu\right|_{\partial \Omega}=0 .
\end{array}\right.
$$

As the space for the pressure, we set

$$
\begin{aligned}
\hat{W}_{p}^{1}(\Omega) & =\left\{\pi \in L_{p, \operatorname{loc}}(\bar{\Omega}) \mid \nabla \pi \in L_{p}(\Omega)^{n}\right\}, \\
X_{p}(\Omega) & =\left\{\pi \in \hat{W}_{p}^{1}(\Omega) \mid\|\pi\|_{X_{p}(\Omega)}<\infty\right\} .
\end{aligned}
$$

When $\Omega$ is a bounded domain, $\|\pi\|_{X_{p}(\Omega)}=\|\pi\|_{W_{p}^{1}(\Omega)}$ and $W_{p}^{1}(\Omega)=X_{p}(\Omega)$. When $\Omega$ is an exterior domain,

$$
\begin{aligned}
\|\pi\|_{X_{p}(\Omega)} & = \begin{cases}\|\nabla \pi\|_{L_{p}(\Omega)}+\|\pi / d\|_{L_{p}(\Omega)}, & n \leq p<\infty, \\
\|\nabla \pi\|_{L_{p}(\Omega)}+\|\pi / d\|_{L_{p}(\Omega)}+\|\pi\|_{L_{\frac{n p}{n-p}(\Omega)}}, & 1<p<n,\end{cases} \\
d(x) & = \begin{cases}2+|x|, & p \neq n, \\
(2+|x|) \log (2+|x|), & p=n .\end{cases}
\end{aligned}
$$

Concerning (1.1), we have the following theorem proved by Shibata and Shimizu [15], which is the base of our analytic semigroup approach to (1.1).

TheOREM 2.1. Let $1<p<\infty, 0<\epsilon<\pi / 2$ and $\delta>0$. We set

$$
\Sigma_{\epsilon}=\{\lambda \in \mathbb{C} \backslash\{0\}|| \arg \lambda \mid \leq \pi-\epsilon\} .
$$


For every $\lambda \in \Sigma_{\epsilon}$ and $f \in L_{p}(\Omega)^{n}$, there exists a unique solution $(u, \pi) \in W_{p}^{2}(\Omega)^{n} \times X_{p}(\Omega)$ of (1). Moreover, the $(u, \pi)$ satisfies the estimate:

$$
|\lambda|\|u\|_{L_{p}(\Omega)}+\|u\|_{W_{p}^{2}(\Omega)}+\|\pi\|_{X_{p}(\Omega)} \leq C_{\epsilon, \delta, p}\|f\|_{L_{p}(\Omega)}
$$

for any $\lambda \in \Sigma_{\epsilon}$ with $|\lambda| \geq \delta$.

3. Analytic semigroup approach to (1.1). In order to formulate (1.1) in the analytic semigroup framework, first of all we have to introduce the 2nd Helmholtz decomposition:

$$
L_{p}(\Omega)^{n}=J_{p}(\Omega) \oplus G_{p}(\Omega)
$$

where we have set

$$
\begin{aligned}
& J_{p}(\Omega)=\left\{u \in L_{p}(\Omega)^{n} \mid \nabla \cdot u=0 \quad \text { in } \Omega\right\}, \\
& G_{p}(\Omega)=\left\{\nabla \pi \mid \pi \in \dot{X}_{p}(\Omega)\right\}, \\
& \dot{X}_{p}(\Omega)=\left\{\pi \in X_{p}(\Omega)|\pi|_{\partial \Omega}=0\right\} .
\end{aligned}
$$

To prove the 2nd Helmholtz decomposition and also the unique solvability of the Laplace equation with Dirichlet condition, we use the following theorem which is proved by letting $\lambda \rightarrow \infty$ in (2.1) and using Theorem 2.1.

LEMmA 3.1. (A) Given $f \in L_{p}(\Omega)^{n}$, there exist unique $g \in J_{p}(\Omega)$ and $\pi \in \dot{X}_{p}(\Omega)$ such that $f=g+\nabla \pi$ in $\Omega$.

(B) If $\pi \in \hat{W}_{p}^{1}(\Omega)$ satisfies $\Delta \pi=0$ in $\Omega$ and $\left.\pi\right|_{\partial \Omega}=0$, then $\pi=0$.

(C) Given $h \in W_{p}^{1-1 / p}(\partial \Omega)$, there exists a $\pi \in X_{p}(\Omega)$ which solves the equation:

$$
\Delta \pi=0 \quad \text { in } \Omega,\left.\quad \pi\right|_{\partial \Omega}=h .
$$

Let $P_{p}: L_{p}(\Omega)^{n} \rightarrow J_{p}(\Omega)$ be the solenoidal projection, and then there exists a unique $\theta \in \dot{X}_{p}(\Omega)$ such that $f=P_{p} f+\nabla \theta$. Inserting this formula into (2.1) and noting that $\left.\theta\right|_{\partial \Omega}=0,(2.1)$ is reduced to the equation:

$$
\begin{aligned}
& \lambda u-\Delta u+\nabla(\pi-\theta)=P_{p} f, \quad \operatorname{div} u=0 \quad \text { in } \Omega, \\
& \left.\mathrm{T}(u, \pi-\theta) \nu\right|_{\partial \Omega}=0 .
\end{aligned}
$$

Therefore we consider (2.1) for $f \in J_{p}(\Omega)$, below.

Now, we shall introduce the reduced Stokes equation corresponding to (2.1). Given $f \in J_{p}(\Omega)$, let $(u, \pi) \in W_{p}^{2}(\Omega)^{n} \times X_{p}(\Omega)$ be a solution of the equation:

$$
\begin{aligned}
& \lambda u-\Delta u+\nabla \pi=f, \quad \nabla \cdot u=0 \text { in } \Omega \\
& \left.(\mathrm{T}(u, \pi) \nu)_{i}\right|_{\partial \Omega}=\sum_{j=1}^{n} \nu_{j}\left(\partial_{j} u_{i}+\partial_{i} u_{j}\right)-\left.\nu_{i} \pi\right|_{\partial \Omega}=0(i=1, \ldots, n),
\end{aligned}
$$

where $(\mathrm{T}(u, \pi) \nu)_{i}$ denotes the $i$-th component of the $n$-vector $\mathrm{T}(u, \pi) \nu$. Applying the divergence to the first equation implies that $\Delta \pi=0$ in $\Omega$. Multiplying the boundary condition by $\nu_{i}$ and using $\sum_{i=1}^{n} \nu_{i}^{2}=1$ on $\partial \Omega$ and $\operatorname{div} u=0$ in $\Omega$, we have

$$
\left.\pi\right|_{\partial \Omega}=\sum_{i, j=1}^{n} \nu_{i} \nu_{j} D_{i j}(u)-\left.\operatorname{div} u\right|_{\partial \Omega} .
$$


In view of Lemma 3.1, there exists a solution operator $K: W_{p}^{1-1 / p}(\partial \Omega)^{n} \rightarrow X_{p}(\Omega)$ associated with the equation:

$$
\Delta K(u)=0 \text { in } \Omega,\left.\quad K(u)\right|_{\partial \Omega}=\sum_{i, j=1}^{n} \nu_{i} \nu_{j} D_{i j}(u)-\left.\operatorname{div} u\right|_{\partial \Omega}
$$

such that there holds the estimate:

$$
\|K(u)\|_{X_{p}(\Omega)} \leq C_{p}\|u\|_{W_{p}^{1-1 / p}(\partial \Omega)} .
$$

Using the operator $K$, we see that when $f \in J_{p}(\Omega)$, the problem:

$$
\begin{aligned}
& \lambda u-\Delta u+\nabla \pi=f, \quad \nabla \cdot u=0 \text { in } \Omega, \\
& \sum_{j=1}^{n} \nu_{j}\left(\partial_{j} u_{i}+\partial_{i} u_{j}\right)-\left.\nu_{i} \pi\right|_{\partial \Omega}=0(i=1, \ldots, n)
\end{aligned}
$$

is equivalent to the reduced Stokes resolvent problem

$$
\begin{aligned}
& \lambda u-\Delta u+\nabla K(u)=f \quad \text { in } \Omega, \\
& \left.\mathrm{T}(u, K(u)) \nu\right|_{\partial \Omega}=0 .
\end{aligned}
$$

The reason why we insert $\operatorname{div} u$ into the boundary condition is to prove that the solution $u$ of (3.1) satisfies the condition: $\operatorname{div} u=0$ in $\Omega$. Theorem 2.1 implies the following theorem immediately.

Theorem 3.2. Let $1<p<\infty, 0<\epsilon<\pi / 2$ and $\delta>0$. Given $\lambda \in \Sigma_{\epsilon}$ and $f \in L_{p}(\Omega)^{n}$, (3.1) admits a unique solution $u \in W_{p}^{2}(\Omega)^{n}$ satisfying the estimate:

$$
|\lambda|\|u\|_{L_{p}(\Omega)}+\|u\|_{W_{p}^{2}(\Omega)} \leq C_{\epsilon, \delta, p}\|f\|_{L_{p}(\Omega)}
$$

for any $\lambda \in \Sigma_{\epsilon}$ with $|\lambda| \geq \delta$.

Let us define the reduced Stokes operator $A_{p}$ by the relations:

$$
\begin{aligned}
A_{p} u & =-\Delta u+\nabla K(u) \quad \text { for } u \in \mathcal{D}\left(A_{p}\right), \\
\mathcal{D}\left(A_{p}\right) & =\left\{u \in J_{p}(\Omega) \cap W_{p}^{2}(\Omega)^{n}|\mathrm{~T}(u, K(u)) \nu|_{\partial \Omega}=0\right\} .
\end{aligned}
$$

Then (3.1) is formulated as $\lambda u+A_{p} u=f$ in $\Omega$ and $u \in \mathcal{D}\left(A_{p}\right)$. Letting $\lambda \rightarrow \infty$ in (3.1), by Theorem 3.2 we obtain the following lemma.

Lemma 3.3. Let $1<p<\infty$. Then, $A_{p}$ is a densely defined closed operator.

Combining Theorem 3.2 and Lemma 3.3, we obtain the following theorem.

Theorem 3.4. Let $1<p<\infty$. Then, $A_{p}$ generates an analytic semigroup $\{T(t)\}_{t \geq 0}$ on $J_{p}(\Omega)$.

Moreover, we can also prove the following theorem concerning the dual space and the adjoint operator.

Theorem 3.5. Let $1<p<\infty$ and $p^{\prime}=p /(p-1)$. Then, $J_{p}(\Omega)^{*}=J_{p^{\prime}}(\Omega)$ and $A_{p}^{*}=A_{p^{\prime}}$. 


\section{4. $L_{p}-L_{q}$ estimate of $(1.1)$}

4.1. The bounded domain case. Let $\Omega$ be a $C^{2,1}$-class bounded domain in $\mathbb{R}^{n}(n \geq 2)$. Let us set

$$
\mathcal{R}=\left\{A x+b \mid A \text { is an anti-symmetric matrix and } b \in \mathbb{R}^{n}\right\} .
$$

Let $p_{1}, \ldots, p_{M}(M=n(n-1) / 2+n)$ be an orthogonal basis of $\mathcal{R}$ in $\Omega$ such that $\left(p_{j}, p_{k}\right)_{\Omega}=\delta_{j k}$. Let us set

$$
\dot{L}_{p}(\Omega)=\left\{u \in L_{p}(\Omega)^{n} \mid\left(u, p_{k}\right)_{\Omega}=0, \quad k=1, \ldots, M\right\} .
$$

Then, we have the following exponential stability of the semigroup $\{T(t)\}_{t \geq 0}$ in the bounded domain case.

THEOREM 4.1. Given any $f \in J_{p}(\Omega) \cap \dot{L}_{p}(\Omega)$, we have

$$
\left\|\nabla^{j} T(t) f\right\|_{L_{q}(\Omega)} \leq C_{p, q} e^{-c t} t^{-\frac{n}{2}\left(\frac{1}{p}-\frac{1}{q}\right)-\frac{j}{2}}\|f\|_{L_{p}(\Omega)}
$$

for $1 \leq p \leq q \leq \infty(p \neq \infty, q \neq 1), t>0$ and $j=0,1$, where $c=c_{p, q}$ is a positive constant.

To prove this theorem, the key is the solvability of the following problem:

$$
\begin{aligned}
-\operatorname{Div} \mathrm{T}(u, \pi) & =f, \quad \operatorname{div} u=0 \text { in } \Omega, \\
\left.\mathrm{T}(u, \pi) \nu\right|_{\partial \Omega} & =g .
\end{aligned}
$$

In fact, we have the following theorem concerning this equation.

TheOREM 4.2. Let $1<p<\infty$. Given $f \in L_{p}(\Omega)^{n}$ and $g \in W_{p}^{1-1 / p}(\partial \Omega)^{n}$ satisfying the condition:

$$
\left(f, p_{j}\right)_{\Omega}+\left(g, p_{j}\right)_{\partial \Omega}=0, \quad j=1, \ldots, M,
$$

(4.1) admits a unique solution

$$
(u, \pi) \in\left(W_{p}^{2}(\Omega)^{n} \cap \dot{L}_{p}(\Omega)\right) \times W_{p}^{1}(\Omega) .
$$

Combining this theorem with Theorem 3.2, we have the following theorem.

TheOREM 4.3. Let $1<p<\infty$ and $0<\epsilon<\pi / 2$. Then, there exists a $\sigma>0$ such that given $f \in J_{p}(\Omega) \cap \dot{L}_{p}(\Omega)$ and $\lambda \in \Sigma_{\epsilon} \cup\{\lambda \in \mathbb{C}|| \lambda \mid \leq \sigma\}$, we have

$$
|\lambda|\left\|\left(\lambda+A_{p}\right)^{-1} f\right\|_{L_{p}(\Omega)}+\left\|\left(\lambda+A_{p}\right)^{-1} f\right\|_{W_{p}^{2}(\Omega)} \leq C_{p}\|f\|_{L_{p}(\Omega)} .
$$

By Theorem 4.3, we have immediately

$$
\|T(t) f\|_{W_{p}^{j}(\Omega)} \leq C_{p} e^{-c t} t^{-\frac{j}{2}}\|f\|_{L_{p}(\Omega)}, \quad j=0,2 .
$$

By using the complex interpolation:

$$
\left(L_{p}(\Omega), W_{p}^{2}(\Omega)\right)_{\theta}=W_{p}^{s}(\Omega), \quad \theta=s / 2,
$$

the real interpolation:

$$
\left[L_{p}(\Omega), W_{p}^{2}(\Omega)\right]_{\theta, 1}=B_{p, 1}^{n / p}(\Omega), \quad \theta=n / 2 p,
$$


the embedding theorems:

$$
\begin{aligned}
& W_{p}^{s}(\Omega) \subset L_{q}(\Omega), s=n\left(\frac{1}{p}-\frac{1}{q}\right)(q \neq \infty), \\
& B_{p, 1}^{n / p}(\Omega) \subset L_{\infty}(\Omega),
\end{aligned}
$$

the semigroup property: $T(t) f=T(t / 2) T(t / 2) f$ and the dual argument, we can show Theorem 4.1 from (4.2).

4.2. The exterior domain case. Let $\Omega$ be an exterior domain in $\mathbb{R}^{n}(n \geq 3)$, whose boundary $\partial \Omega$ is a $C^{2,1}$ hypersurface. Then, we have the following theorem.

THEOREM 4.4 .

$$
\|T(t) f\|_{L_{q}(\Omega)} \leq C_{p, q} t^{-\frac{n}{2}\left(\frac{1}{p}-\frac{1}{q}\right)}\|f\|_{L_{p}(\Omega)}
$$

for $1 \leq p \leq q \leq \infty(p \neq \infty, q \neq 1), t>0$ and $f \in J_{p}(\Omega)$, and

$$
\|\nabla T(t) f\|_{L_{q}(\Omega)} \leq C_{p, q} t^{-\frac{n}{2}\left(\frac{1}{p}-\frac{1}{q}\right)-\frac{1}{2}}\|f\|_{L_{p}(\Omega)}
$$

for $1 \leq p \leq q \leq \infty(p \neq \infty, q \neq 1), t>0$ and $f \in J_{p}(\Omega)$.

REMARK 4.5. If we consider the non-slip boundary condition $\left.u\right|_{\partial \Omega}=0$ instead of the Neumann boundary condition, to obtain (4.4) we have to assume that $1 \leq p \leq q \leq n$ $(q \neq 1)$ (cf. [11], [12], [14], [4], [5] and [6]).

\section{A sketch of proof of Theorem 4.4}

5.1. 1st step. Construction of a solution operator $R(\lambda)$. The following theorem is concerned with the solution operator to (2.1).

THEOREM 5.1. Let $1<p \leq q \leq \infty$ and set

$$
L_{p, R}(\Omega)=\left\{f \in L_{p}(\Omega)^{n} \mid f(x)=0 \quad x \notin B_{R}\right\} .
$$

Then, there exists an $\epsilon>0$ and an operator $R(\lambda)=\left(R_{0}(\lambda), R_{1}(\lambda)\right)$ for $\lambda \in \dot{U}_{\epsilon}=\{\lambda \epsilon$ $\mathbb{C} \backslash(-\infty, 0]|| \lambda \mid<\epsilon\}$ having the following properties:

(1) If we set $u=R_{0}(\lambda) f$ and $\pi=R_{1}(\lambda) f$, then $(u, \pi)$ solves the problem:

$$
\lambda u-\operatorname{Div} \mathrm{T}(u, \pi)=f, \quad \operatorname{div} u=0 \quad \text { in } \Omega,\left.\quad \mathrm{T}(u, \pi) \nu\right|_{\partial \Omega}=0 .
$$

(2) There holds the relation: $R_{0}(\lambda) f=(\lambda+A)^{-1} P_{p} f$ for any $\lambda \in \dot{U}_{\epsilon}$ and $f \in L_{p, R}(\Omega)$.

(3) There holds the estimate:

$$
\left\|R_{0}(\lambda) f\right\|_{L_{q}(\Omega)} \leq C_{p, q}|\lambda|^{\frac{n}{2}\left(\frac{1}{p}-\frac{1}{q}\right)-1}\|f\|_{L_{p}(\Omega)}
$$

for $1<p \leq q \leq \infty, \lambda \in \dot{U}_{\epsilon}$ and $f \in L_{p, R}(\Omega)$.

(4) There holds the estimate:

$$
\left\|\nabla R_{0}(\lambda) f\right\|_{L_{p}(\Omega)} \leq C_{p}|\lambda|^{-\min \left(\frac{1}{2}, \frac{n}{2 p}\right)}\|f\|_{L_{p}(\Omega)}
$$

for $\lambda \in \dot{U}_{\epsilon}$ and $f \in L_{p, R}(\Omega)$. 
(5) There holds the expansion formula:

$$
R(\lambda)=\lambda^{\frac{n}{2}-1}(\log \lambda)^{\sigma(n)} H_{0}+\lambda^{\frac{n}{2}-1} H_{1}(\lambda)+H_{2}(\lambda)
$$

for $\lambda \in \dot{U}_{\epsilon}$ on $\Omega_{R}=\Omega \cap B_{R}$,

where

$$
\begin{aligned}
& \sigma(n)=1(n \geq 4, \text { even }), \sigma(n)=0(n \geq 3, \text { odd }) ; \\
& H_{0} \in \mathcal{L}\left(L_{p, R}(\Omega), W_{p}^{2}\left(\Omega_{R}\right)^{n} \times W_{p}^{1}\left(\Omega_{R}\right)\right) ; \\
& H_{1}(\lambda) \in \mathcal{B} \mathcal{A}\left(\dot{U}_{\epsilon}, \mathcal{L}\left(L_{p, R}(\Omega), W_{p}^{2}\left(\Omega_{R}\right)^{n} \times W_{p}^{1}\left(\Omega_{R}\right)\right)\right) ; \\
& H_{2}(\lambda) \in \mathcal{B} \mathcal{A}\left(U_{\epsilon}, \mathcal{L}\left(L_{p, R}(\Omega), W_{p}^{2}\left(\Omega_{R}\right)^{n} \times W_{p}^{1}\left(\Omega_{R}\right)\right)\right) ; \\
& U_{\epsilon}=\{\lambda \in \mathbb{C}|| \lambda \mid<\epsilon\},
\end{aligned}
$$

and $\mathcal{B A}(U, W)$ is the set of all bounded analytic functions on $U$ with their values in $W$.

Using Theorem 5.1, we can show (4.3) and also (4.4) under the assumption: $1 \leq p \leq$ $q \leq n(q \neq 1)$ in Theorem 4.4. To prove Theorem 5.1, we use the solution operator $\left(E_{\lambda}, \Pi\right)$ of the Stokes resolvent equation in $\mathbb{R}^{n}$, which gives the solutions $u=E_{\lambda} f$ and $\pi=\Pi f$ of the equation:

$$
(\lambda-\Delta) u+\nabla \pi=f, \quad \operatorname{div} u=0 \text { in } \mathbb{R}^{n} .
$$

Since $E_{\lambda} f$ is given by the modified Bessel function of order $(n-2) / 2$, applying the Young inequality we have

$$
\left\|\nabla^{j} E_{\lambda} f\right\|_{L_{q}\left(\mathbb{R}^{n}\right)} \leq C_{p, q}|\lambda|^{\frac{n}{2}\left(\frac{1}{p}-\frac{1}{q}\right)-1+\frac{j}{2}}\|f\|_{L_{p}\left(\mathbb{R}^{n}\right)} \quad j=0,1
$$

for $1<p \leq q \leq \infty(p \neq \infty, q \neq 1), \lambda \in \Sigma_{\epsilon}=\{\lambda \in \mathbb{C} \backslash\{0\}|| \lambda \mid \leq \pi-\epsilon\}$ and $f \in L_{p}\left(\mathbb{R}^{n}\right)$. By using the expansion formula of the modified Bessel function near the origin, we have

$$
E_{\lambda} f=\lambda^{\frac{n}{2}-1}(\log \lambda)^{\sigma(n)} G_{1}(\lambda) f+G_{2}(\lambda) f \text { in } B_{R}
$$

for $f \in L_{p, R}\left(\mathbb{R}^{n}\right)=\left\{f \in L_{p}\left(\mathbb{R}^{n}\right)^{n} \mid f(x)=0\right.$ for $\left.x \notin B_{R}\right\}$ and $\lambda \in \dot{U}_{\frac{1}{2}}$, where

$$
G_{j}(\lambda) \in \mathcal{B} \mathcal{A}\left(U_{\frac{1}{2}}, \mathcal{L}\left(L_{p, R}\left(\mathbb{R}^{n}\right), W_{p}^{2}\left(B_{R}\right)\right)\right) .
$$

And also, we use the solution operator $(A, B)$ which gives solutions $u=A f$ and $\pi=B f$ of the interior problem:

$$
\begin{aligned}
& -\operatorname{Div} \mathrm{T}(u, \pi)=f, \quad \operatorname{div} u=0 \text { in } \Omega_{R}, \\
& \left.\mathrm{~T}(u, \pi) \nu\right|_{\partial \Omega}=0, \\
& \left.\mathrm{~T}(u, \pi) \nu_{0}\right|_{S_{R}}=\left.\mathrm{T}\left(E_{0} f_{0}, \Pi f_{0}\right) \nu_{0}\right|_{S_{R}},
\end{aligned}
$$

where $\nu_{0}=x /|x|, S_{R}=\{|x|=R\}, \Omega_{R}=\Omega \cap B_{R}, \partial \Omega_{R}=\partial \Omega \cup S_{R}, f_{0}=f(x \in \Omega)$ and $f_{0}=0(x \notin \Omega)$. Since there holds the compatibility condition:

$$
\left(f, p_{j}\right)_{\Omega_{R}}+\left(\mathrm{T}\left(E_{0} f_{0}, \Pi f_{0}\right) \nu_{0}, p_{j}\right)_{S_{R}}=0
$$

for $j=1, \ldots, M$, we can find $A$ and $B$. Moreover, since $D\left(p_{j}\right)=0$ and $\operatorname{div} p_{j}=0$, we may assume that

$$
\left(A f-E_{0} f_{0}, p_{j}\right)_{\Omega_{R}}=0, j=1, \ldots, M
$$


To define our parametrix for (2.1), we choose a cut-off function $\varphi$ in such a way that

$$
0 \leq \varphi \leq 1, \varphi(x)=1(|x| \leq R-2), \quad \varphi(x)=0(|x| \geq R-1)
$$

where $R$ is a number such that $B_{R} \supset \Omega^{c}$. As the parametrix for (2.1), we set

$$
\begin{aligned}
\Phi_{\lambda} f & =(1-\varphi) E_{\lambda} f_{0}+\varphi A f+\mathbb{B}\left[(\nabla \varphi)\left(E_{\lambda} f-A f\right)\right], \\
\Psi f & =(1-\varphi) \Pi f_{0}+\varphi B f,
\end{aligned}
$$

where $\mathbb{B}$ is the usual Bogovskiı operator (cf. [2], [3], [13], [7]). Then, there exists a compact operator $T_{\lambda}$ of $L_{p, R}(\Omega)$ such that

$$
\begin{aligned}
& \lambda \Phi_{\lambda} f-\operatorname{Div} \mathrm{T}\left(\Phi_{\lambda} f_{0}, \Psi f\right)=\left(I+T_{\lambda}\right) f, \quad \operatorname{div} \Phi_{\lambda} f=0 \text { in } \Omega, \\
& \left.T\left(\Phi_{\lambda} f, \Psi f\right) \nu\right|_{\partial \Omega}=0 .
\end{aligned}
$$

The uniqueness of the solution to the homogeneous equation:

$$
-\operatorname{Div} \mathrm{T}(u, \pi)=0, \operatorname{div} u=0 \text { in } \Omega,\left.\quad \mathrm{T}(u, \pi) \nu\right|_{\partial \Omega}=0
$$

in the class of functions satisfying the radiation condition:

$$
\begin{aligned}
& u(x)=O\left(|x|^{-(n-2)}\right), \nabla u(x)=O\left(|x|^{-(n-1)}\right), \\
& \pi(x)=O\left(|x|^{-(n-1)}\right) \quad \text { as }|x| \rightarrow \infty,
\end{aligned}
$$

and Fredholm's alternative theorem imply the existence of the inverse operator:

$$
\left(I+T_{\lambda}\right)^{-1} \in \mathcal{B} \mathcal{A}\left(\dot{U}_{\epsilon}, \mathcal{L}\left(L_{p, R}(\Omega)\right)\right) .
$$

Therefore, we can define $R(\lambda)$ by the relations:

$$
R_{0}(\lambda)=\Phi_{\lambda}\left(I+T_{\lambda}\right)^{-1}, \quad R_{1}(\lambda)=\Psi\left(I+T_{\lambda}\right)^{-1} .
$$

By this, (5.1) and (5.2), we can show Theorem 5.1.

5.2. 2nd step. Modification of $R(\lambda)$. By using the special structure of Neumann boundary condition, we modify $R(\lambda)$ to prove Theorem 4.4, especially (4.4). In order to do this, we use the following reduction: Given $f \in L_{p}(\Omega)^{n}$, let $u$ and $\pi$ be solutions to the resolvent problem:

$$
\begin{aligned}
& \lambda u-\operatorname{Div} \mathrm{T}(u, \pi)=f, \operatorname{div} u=0 \text { in } \Omega, \\
& \left.\mathrm{T}(u, \pi) \nu\right|_{\partial \Omega}=0 .
\end{aligned}
$$

We set

$$
u=E_{\lambda} f_{0}+v \text { and } \pi=\Pi f_{0}+\theta .
$$

Then, $v$ and $\theta$ enjoy the equation:

$$
\begin{aligned}
& \lambda v-\operatorname{Div} \mathrm{T}(v, \theta)=0, \quad \operatorname{div} v=0 \text { in } \Omega, \\
& \left.\mathrm{T}(v, \theta) \nu\right|_{\partial \Omega}=-\left.\mathrm{T}\left(E_{\lambda} f_{0}, \Pi f_{0}\right) \nu\right|_{\partial \Omega} .
\end{aligned}
$$

Since

$$
\left(\mathrm{T}\left(E_{\lambda} f_{0}, \Pi f_{0}\right) \nu, p_{j}\right)_{\partial \Omega}=-\left(\operatorname{Div} \mathrm{T}\left(E_{\lambda} f_{0}, \Pi f_{0}\right), p_{j}\right)_{\Omega^{c}}=-\left(\lambda E_{\lambda} f_{0}, p_{j}\right)_{\Omega^{c}}
$$


for $j=1, \ldots, M$, there exists $(w, \tau)$ which solves the equation:

$$
\begin{aligned}
& \lambda w-\operatorname{Div} \mathrm{T}(w, \tau)=g_{\lambda}, \quad \operatorname{div} w=0 \text { in } \Omega_{R}, \\
& \left.\mathrm{~T}(w, \tau) \nu\right|_{\partial \Omega}=-\left.\mathrm{T}\left(E_{\lambda} f_{0}, \Pi f_{0}\right) \nu\right|_{\partial \Omega}, \\
& \left.\mathrm{T}(w, \tau) \nu_{0}\right|_{S_{R}}=0,
\end{aligned}
$$

where

$$
g_{\lambda}=\sum_{j=1}^{M}\left(\lambda E_{\lambda} f_{0}, p_{j}\right)_{\Omega^{c}} p_{j}
$$

We set

$$
v=\varphi w+z-\mathbb{B}[(\nabla \cdot \varphi) w] \text { and } \theta=\varphi \tau+\omega,
$$

and then $z$ and $\omega$ enjoy the equation:

$$
\lambda z-\operatorname{Div} \mathrm{T}(z, \omega)=h_{\lambda}, \quad \operatorname{div} z=0 \text { in } \Omega,\left.\quad \mathrm{T}(z, \omega) \nu\right|_{\partial \Omega}=0,
$$

where

$$
\begin{aligned}
h_{\lambda}= & -\varphi g_{\lambda}+2(\nabla \varphi): \nabla w+(\Delta \varphi) w \\
& -\lambda \mathbb{B}[(\nabla \varphi) \cdot w]+\operatorname{Div} D(\mathbb{B}[(\nabla \varphi) \cdot w])-(\nabla \varphi) \tau .
\end{aligned}
$$

We can divide $h_{\lambda}$ into two parts : $h_{\lambda}=h_{\lambda}^{1}+\lambda h_{\lambda}^{2}$, where

$$
\begin{aligned}
& \operatorname{supp}_{\lambda} h_{\lambda}^{j} \subset D_{R-2, R-1}=\left\{x \in \mathbb{R}^{n}|R-2 \leq| x \mid \leq R-1\right\}, \\
& \left(h_{\lambda}^{1}, p_{j}\right)_{\mathbb{R}^{n}}=0, \quad j=1, \ldots, M .
\end{aligned}
$$

Finally, we set

$$
z=z^{1}+\lambda R_{0}(\lambda) h_{\lambda}^{2} \text { and } \omega=\omega^{1}+\lambda R_{1}(\lambda) h_{\lambda}^{2},
$$

and then $z^{1}$ and $\omega^{1}$ enjoy the equation:

$$
\lambda z^{1}-\operatorname{Div} \mathrm{T}\left(z^{1}, \omega^{1}\right)=h_{\lambda}^{1}, \quad \operatorname{div} z^{1}=0 \text { in } \Omega,\left.\quad \mathrm{T}\left(z^{1}, \omega^{1}\right) \nu\right|_{\partial \Omega}=0 .
$$

Now, let us set

$$
\mathcal{I}=\left\{f \in L_{p}\left(\mathbb{R}^{n}\right)^{n} \mid \operatorname{supp} f \subset D_{R-2, R-1},\left(f, p_{j}\right)_{\mathbb{R}^{n}}=0(j=1, \ldots, M)\right\} .
$$

Since $h_{\lambda}^{1} \in \mathcal{I}$, we consider the problem :

$$
\begin{aligned}
& \lambda u-\operatorname{Div} \mathrm{T}(u, \pi)=f, \quad \operatorname{div} u=0 \text { in } \Omega, \\
& \left.\mathrm{T}(u, \pi) \nu\right|_{\partial \Omega}=0
\end{aligned}
$$

with $f \in \mathcal{I}$. Recall that

$$
\begin{aligned}
& \lambda \Phi_{\lambda} f-\operatorname{Div} \mathrm{T}\left(\Phi_{\lambda} f_{0}, \Psi f\right)=\left(I+T_{\lambda}\right) f, \quad \operatorname{div} \Phi_{\lambda} f=0 \text { in } \Omega, \\
& \left.T\left(\Phi_{\lambda} f, \Psi f\right) \nu\right|_{\partial \Omega}=0 .
\end{aligned}
$$

The point is that we can divide $T_{\lambda}$ into two parts: $T_{\lambda}=A_{\lambda}+\lambda B_{\lambda}$, where

$A_{\lambda}$ is a compact operator on $\mathcal{I}$;

$\left\|A_{\lambda} f-A_{0} f\right\|_{L_{p}} \leq C|\lambda|^{1 / 2}\|f\|_{L_{p}}$

$B_{\lambda}$ is a bounded operator from $\mathcal{I}$ into $L_{p, R}(\Omega)$. 
Therefore, if we set

$$
\begin{aligned}
& U_{\lambda} f=\Phi_{\lambda} f-\lambda R_{0}(\lambda) B_{\lambda} f, \\
& \Theta_{\lambda} f=\Psi f-\lambda R_{1}(\lambda) B_{\lambda} f,
\end{aligned}
$$

then we see that

$$
\begin{aligned}
& \lambda U_{\lambda} f-\operatorname{Div} \mathrm{T}\left(U_{\lambda} f, \Theta_{\lambda} f\right)=f+A_{\lambda} f, \quad \operatorname{div} U_{\lambda} f=0 \text { in } \Omega, \\
& \left.\mathrm{T}\left(U_{\lambda} f, \Theta_{\lambda} f\right) \nu\right|_{\partial \Omega}=0 .
\end{aligned}
$$

By using the uniqueness of the solution to the Stokes equation with Neumann boundary condition and the Fredholm alternative theorem, we can show that there exists an $\epsilon>0$ such that

$$
\left(I+A_{\lambda}\right)^{-1} \in \mathcal{B} \mathcal{A}\left(\dot{U}_{\epsilon}, \mathcal{L}(\mathcal{I})\right)
$$

From these consideration, by using not only (5.1) and Theorem 5.1 but also the relation:

$$
E_{\lambda} f=\lambda^{\frac{n}{2}}(\log \lambda)^{\sigma(n)} G_{1}^{\prime}(\lambda) f+G_{2}(\lambda) f, \quad f \in \mathcal{I},
$$

on $B_{R}$ with some $G_{1}^{\prime}(\lambda) \in \mathcal{B} \mathcal{A}\left(U_{1 / 2}, \mathcal{L}\left(\mathcal{I}, W_{p}^{2}\left(B_{R}\right)^{n} \times W_{p}^{1}\left(B_{R}\right)\right)\right)$, we can show the following proposition.

Proposition 5.2. There exist operators $Y(\lambda)$ and $Z(\lambda)$ such that for any $f \in L_{p}(\Omega)^{n}$

$$
\begin{aligned}
& (\lambda+A)^{-1} P_{p} f=Y(\lambda) f+Z(\lambda) f, \quad \lambda \in \Sigma_{\epsilon} \cap U_{\epsilon}, \\
& \|Y(\lambda) f\|_{L_{q}(\Omega)} \leq C_{p, q}|\lambda|^{\frac{n}{2}\left(\frac{1}{p}-\frac{1}{q}\right)-1}\|f\|_{L_{p}(\Omega)}, \\
& \|\nabla Y(\lambda) f\|_{L_{q}(\Omega)} \leq C_{p, q}|\lambda|^{\frac{n}{2}\left(\frac{1}{p}-\frac{1}{q}\right)-\frac{1}{2}}\|f\|_{L_{p}(\Omega)},
\end{aligned}
$$

for any $1<p \leq q \leq \infty(p \neq \infty), \lambda \in \dot{U}_{\epsilon}$ and

$$
Z(\lambda) f \in \mathcal{B} \mathcal{A}\left(U_{\epsilon}, \mathcal{L}\left(L_{p}(\Omega), W_{\infty}^{2}(\Omega)\right)\right), \quad \operatorname{supp} Z(\lambda) f \subset B_{R} .
$$

If we write

$$
T(t) f=\frac{1}{2 \pi} \int_{\Gamma_{1}} e^{\lambda t}(\lambda I+A)^{-1} f d \lambda+\frac{1}{2 \pi} \int_{\Gamma_{2}} e^{\lambda t}(Y(\lambda)+Z(\lambda)) f d \lambda
$$

where

$$
\Gamma_{1}=\left\{s e^{ \pm i \theta_{0}} \mid \epsilon \leq s<\infty\right\}, \quad \frac{\pi}{2}<\theta_{0}<\pi, \quad \Gamma_{2}=\left\{\epsilon e^{i \theta} \mid-\theta_{0} \leq \theta \leq \theta_{0}\right\},
$$

then by Proposition 5.2 and Theorem 3.2 we can show Theorem 4.4.

\section{References}

[1] H. Abels, Stokes equations in asymptotically flat domains and the motion of a free surface, Darmstadt, Techn. Univ. Diss., 2003.

[2] M. E. Bogovskiu, Solution of the first boundary value problem for the equation of continuity of an incompressible medium, Dokl. Akad. Nauk SSSR 248 (1979), 1037-1049; English transl: Soviet Math. Dokl. 20 (1979), 1094-1098.

[3] M. E. Bogovskiǔ, Solution of some vector analysis problems connected with operators div and grad, Trudy Seminara S. L. Soboleva 1 (1980), 5-40 (in Russian). 
[4] W. Dan and Y. Shibata, On the $L_{q}-L_{r}$ estimates of the Stokes semigroup in a two dimensional exterior domain, J. Math. Soc. Japan 51 (1999), 181-207.

[5] W. Dan and Y. Shibata, Remark on the $L_{q}-L_{\infty}$ estimate of the Stokes semigroup in a 2-dimensional exterior domain, Pacific J. Math. 189 (1999), 223-240.

[6] W. Dan, T. Kobayashi and Y. Shibata, On the local energy decay approach to some fluid flow in exterior domain, in: Recent Topics on Mathematical Theory of Viscous Incompressible Fluid, Lecture Notes Numer. Appl. Math. 16, Kinokuniya, Tokyo, 1998, 1-51.

[7] G. P. Galdi, An Introduction to the Mathematical Theory of the Navier-Stokes Equations, Vol I: Linearized Steady Problems, Springer Tracts in Natural Philosophy, 38, SpringerVerlag, New York, 1994.

[8] G. Grubb, Parameter-elliptic and parabolic pseudodifferential boundary problems in global $L_{p}$ Sobolev spaces, Math. Z. 218 (1995), 43-90.

[9] G. Grubb, Nonhomogeneous time-dependent Navier-Stokes problems in $L_{p}$ Sobolev spaces, Differential Integtral Equations 8 (1995), 1013-1046.

[10] G. Grubb and V. A. Solonnikov, Boundary value problems for the nonstationary NavierStokes equations treated by pseudo-differential method, Math. Scand. 69 (1991), 217-290.

[11] H. Iwashita, $L_{q}-L_{r}$ estimates for solutions of the nonstationary Stokes equations in an exterior domain and the Navier-Stokes initial value problems in $L_{p}$ spaces, Math. Ann. 285 (1989), 265-288.

[12] P. Maremonti and V. A. Solonnikov, On nonstationary Stokes problem in exterior domains, Ann. Sc. Norm. Sup. Pisa 24 (1997), 395-449.

[13] K. Pileckas, On spaces of solenoidal vectors Trudy Mat. Inst. Steklov 159 (1983), 137-149; English transl: Steklov Math. Inst. 159 (1984), 141-154.

[14] Y. Shibata, On an exterior initial boundary value problem for Navier-Stokes equations, Quart. Appl. Math. LVII (1999), 117-155.

[15] Y. Shibata and S. Shimizu, On a resolvent estimate for the Stokes system with Neumann boundary condition, Differential Integtral Equations 16 (2003), 385-426.

[16] V. A. Solonnikov, On the transient motion of an isolated volume of viscous incompressible fluid, Math. USSR Izvestiya 31 (1988), 381-405. 